\title{
The effect of early arm exercise on drainage volume after total mastectomy and tissue expander insertion in breast cancer patients: a prospective study
}

\author{
Oh Young Joo, Seung Jin Moon, Dong Won Lee, Dae Hyun Lew, Won Jai Lee, Seung Yong Song \\ Department of Plastic and Reconstructive Surgery, Institute of Human Tissue Restoration, Severance Hospital, Yonsei University College of \\ Medicine, Seoul, Korea
}

\begin{abstract}
Background In prosthesis-based breast reconstruction patients, the drain tends to be kept in place longer than in patients who undergo only mastectomy. Postoperative arm exercise also increases the drainage volume. However, to preserve shoulder function, early exercise is recommended. In this study, we investigated the effect of early exercise on the total drainage volume and drain duration in these patients.

Methods We designed a prospective randomized trial involving 56 patients who underwent immediate breast reconstruction following mastectomy using tissue expanders. In each group, the patients were randomized either to perform early arm exercises using specific shoulder movement guidelines 2 days after surgery or to restrict arm movement above the shoulder height until drain removal. The drain duration and the total amount of drainage were the primary endpoints.

Results There were no significant differences in age, height, weight, body mass index, or mastectomy specimen weight between the two groups. The total amount of drainage was $1,497 \mathrm{~mL}$ in the early exercise group and $1,336 \mathrm{~mL}$ in the exercise restriction group. The duration until complete removal of the drains was 19.71 days in the early exercise group and 17.11 days in the exercise restriction group.

Conclusions Exercise restriction after breast reconstruction did not lead to a significant difference in the drainage volume or the average time until drain removal. Thus, early exercise is recommended for improved shoulder mobility postoperatively. More long-term studies are needed to determine the effect of early exercise on shoulder mobility in prosthesis-based breast reconstruction patients.
\end{abstract}

Keywords Arm exercise / Mastectomy / Drainage volume / Mammaplasty
Correspondence: Seung Yong Song Department of Plastic and Reconstructive Surgery, Yonsei University College of Medicine, 50-1 Yonsei-ro, Seodaemun-gu, Seoul 03722, Korea

Tel: $+82-2-2228-2214$

Fax: +82-2-393-6947

E-mail: pssysong@yuhs.ac

Received: March 22, 2021 • Revised: June 5, 2021 • Accepted: July 7, 2021

pISSN: 2234-6163 • elSSN: 2234-6171 • https://doi.org/10.5999/aps.2021.00500 • Arch Plast Surg 2021;48:583-589

\section{INTRODUCTION}

Arm and shoulder morbidity post-mastectomy is a common problem for some women following breast cancer surgery [1].
To prevent shoulder joint dysfunction, early arm and shoulder exercise programs have been recommended for these patients. Early exercise is especially emphasized in patients undergoing axillary lymph node dissection (ALND) along with mastecto-

Copyright $\odot 2021$ The Korean Society of Plastic and Reconstructive Surgeons

This is an Open Access article distributed under the terms of the Creative Commons Attribution Non-Commercial License (https://creativecommons.org/

licenses/by-nc/4.0/) which permits unrestricted non-commercial use, distribution, and reproduction in any medium, provided the original work is properly cited. I www.e-aps.org 
my, since they are more likely to experience upper arm movement problems [2]. Early exercise is currently the focus of attention due to its known benefits compared with delayed exercise, such as better mobility in arm flexion and earlier recovery of mobility [3].

In general, a longer duration and a larger volume of drainage are expected in patients who undergo expander-based breast reconstruction than in those who are treated with mastectomy alone. Shoulder exercise itself may also increase the incidence of postoperative seroma, which is among the most common complications of mastectomy [4]. In a systematic review, a longer drainage duration increased the risk of surgical-site infections [5], which may lead to the unwanted removal of tissue expanders. To prevent such complications, some surgeons limit shoulder and arm exercises to certain ranges of motion postoperatively.

Since there is a lack of relevant studies regarding the effects of tissue expander insertion on drainage volume and exercise limitations, patients are generally requested to participate in various arm exercise programs that follow the surgeon's preferences. At our institution, mastectomy-only patients are introduced to exercises that limit movement of the arm below $90^{\circ}$ in all planes of movement starting after postoperative day 2 . An exercise routine is recommended three times a day, which includes raising the arm slowly 10 times until reaching $90^{\circ}$. Full vigorous shoulder movement is recommended after drainage removal. Immediate expander-based reconstruction patients are restricted to only minimal arm exercise until they undergo total drainage removal, which usually takes around 2 weeks.

de Haan et al. [6] noted that expander-based reconstruction patients suffered a functional loss of muscle movement compared to a mastectomy-only comparison group. Therefore, this prospective study was performed to analyze the effect of shoulder exercise on the postoperative drainage volume and also to determine whether delayed exercise is beneficial for ultimately reducing the period of limited arm exercise in breast reconstruction patients.

\section{METHODS}

\section{Patients}

From March 2018 through September 2018, female patients over the age of 20 who underwent expander-based breast reconstruction immediately post-mastectomy were prospectively randomized into two groups after they provided informed consent. Randomization was carried out using a random number table and a block randomization method. Patients with a history of neo-adjuvant chemotherapy, previous surgery or radiotherapy in the ipsilateral axilla area, or recurrent breast cancer were ex- cluded.

This two-group (early vs. delayed shoulder exercise), blockrandomized controlled study received approval from the Institutional Review Board of Severance Hospital (IRB No. 4-20171054) and was carried out in accordance with the principles of the Declaration of Helsinki.

\section{Operative technique}

Patients underwent one of three mastectomy techniques: nipple-sparing mastectomy, skin-sparing mastectomy, or total mastectomy. Mastectomy along with sentinel lymph node biopsy (SLNB) or ALND was performed by an oncologic surgeon. In total, 48 patients received SLNB, and eight patients underwent ALND (Table 1). Tissue expander-based breast reconstruction was performed by a single plastic surgeon using either a Mentor CPX4 expander (Mentor Worldwide LLC, Irvine, CA, USA) or an Allergan expander (Allergan PLC, Dublin, Ireland). The pectoralis major muscle was elevated using a Bovie electrocautery device, and the same type of acellular dermal matrix (ADM) sling was sutured to the inferolateral border of the muscle with a MegaDerm graft $(6 \times 16 \mathrm{~cm}, 1.5 \mathrm{~mm}$ thick; L\&C Bio Corp., Seoul, Korea). This is an allogenic ADM graft obtained from human cadaver skin following an appropriate solvent and detergent process to eliminate any cellular components. Two closed drainage systems were placed in the inframammary fold area and in the axillary space. The drainage volume was checked daily, and the drains were removed without additional procedures (i.e., cutting and advancing) when their output was $<30 \mathrm{~mL} /$ day for two consecutive days.

Table 1. Patient characteristics summarized by exercise group

\begin{tabular}{lrrr}
\hline Variable & $\begin{array}{c}\text { Early exercise } \\
(\mathrm{n}=28)\end{array}$ & $\begin{array}{c}\text { Arm restriction } \\
(\mathrm{n}=28)\end{array}$ & P-value \\
\hline Age $(\mathrm{yr})$ & $44.50 \pm 6.70$ & $44.10 \pm 8.35$ & 0.832 \\
Height $(\mathrm{cm})$ & $160.10 \pm 3.48$ & $159.10 \pm 5.05$ & 0.448 \\
Weight $(\mathrm{kg})$ & $55.00 \pm 8.08$ & $55.50 \pm 8.01$ & 0.845 \\
Body mass index $\left(\mathrm{kg} / \mathrm{m}^{2}\right)$ & $21.40 \pm 2.82$ & $21.90 \pm 2.60$ & 0.481 \\
Mastectomy specimen weight (g) & $357.40 \pm 198.45$ & $381.30 \pm 175.03$ & 0.624 \\
Lymph node surgery & & & 0.126 \\
Sentinel lymph node biopsy & 26 & 22 & \\
Axillary lymph node dissection & 2 & 6 & \\
History & & & \\
$\quad$ Smoking & 0 & 1 & \\
Hypertension & 2 & 2 & \\
Diabetes & 0 & 0 & \\
Type of expander ${ }^{\text {a) }}$ & & & \\
Allergan & 25 & 23 & \\
Mentor & 3 & 5 & \\
\hline
\end{tabular}

Values are presented as mean $\pm \mathrm{SD}$ or number.

${ }^{a}$ T)wo types of expanders were used for all patients. 


\section{Intervention}

After surgery, the patients were provided with printed information about arm exercises in accordance with their assigned group. The early exercise group $(n=28)$ started arm exercises on postoperative day 2 . Each exercise routine was performed three times a day, and included 10 repetitions of the following steps (Fig. 1): (A) abduction of the ipsilateral shoulder joint to $90^{\circ}$, (B) flexion and then extension of the elbow and wrist joints with the hands pointing upwards, (C) horizontal abduction of the shoulder joint, and (D) circumduction of the shoulder joint. In the delayed exercise group $(n=28)$, any type of arm exercise was restricted until all drainage systems were removed. Patients in the early exercise group were asked to actively follow these steps, whereas the delayed exercise patients were advised to observe total restriction of movement in their shoulder joints. For both groups, a full range of shoulder and elbow movement was introduced after drainage removal.

\section{Measurements}

The drainage volume was checked daily until the drain was removed. Each drain was removed when its output was $<50 \mathrm{~mL}$ per 48 hours. The total drainage volume and duration of drain placement were recorded using values from the date of final drain removal.

\section{Statistics}

All statistical analyses were performed using SPSS for Windows version 25.0 (IBM Corp., Armonk, NY, USA). Data are expressed as the mean value \pm standard deviation. The level of significance was set at 0.05 . Univariate analyses were carried out using the Fisher exact test. Linear regression was performed to evaluate the associations of patient characteristics with the total drainage volume and the total drain duration.

\section{RESULTS}

We included 56 patients: 28 in each group. No significant differences were observed in patients' characteristics between both groups at baseline (Table 1). The total drainage volume gradually decreased in a similar pattern for both groups (Fig. 2). The relationship between body mass index (BMI) and the total drainage volume is shown in Fig. 3. In the early exercise program, none of the patients suffered pain that required them to stop exercising. The average total drainage volume was $1,497.00 \pm$ $595.30 \mathrm{~mL}$ for the early exercise group and $1,336.00 \pm 581.82$ $\mathrm{mL}$ in the arm restriction group, which was not a statistically significant difference. No strong correlation $(\mathrm{P}=0.106)$ was found between the two groups regarding the duration of the drainage system (early exercise group, $19.71 \pm 7.04$ days; arm restriction group, $17.11 \pm 4.55$ days) (Table 2 ).

The patients who received ALND were first excluded and then analyzed in the same manner, as shown in Table 3 (early exercise group, $1,457.00 \pm 558.91 \mathrm{~mL}, 19.62 \pm 6.95$ days; arm restriction group, $1,303.00 \pm 596.20 \mathrm{~mL}, 16.64 \pm 4.70$ days,
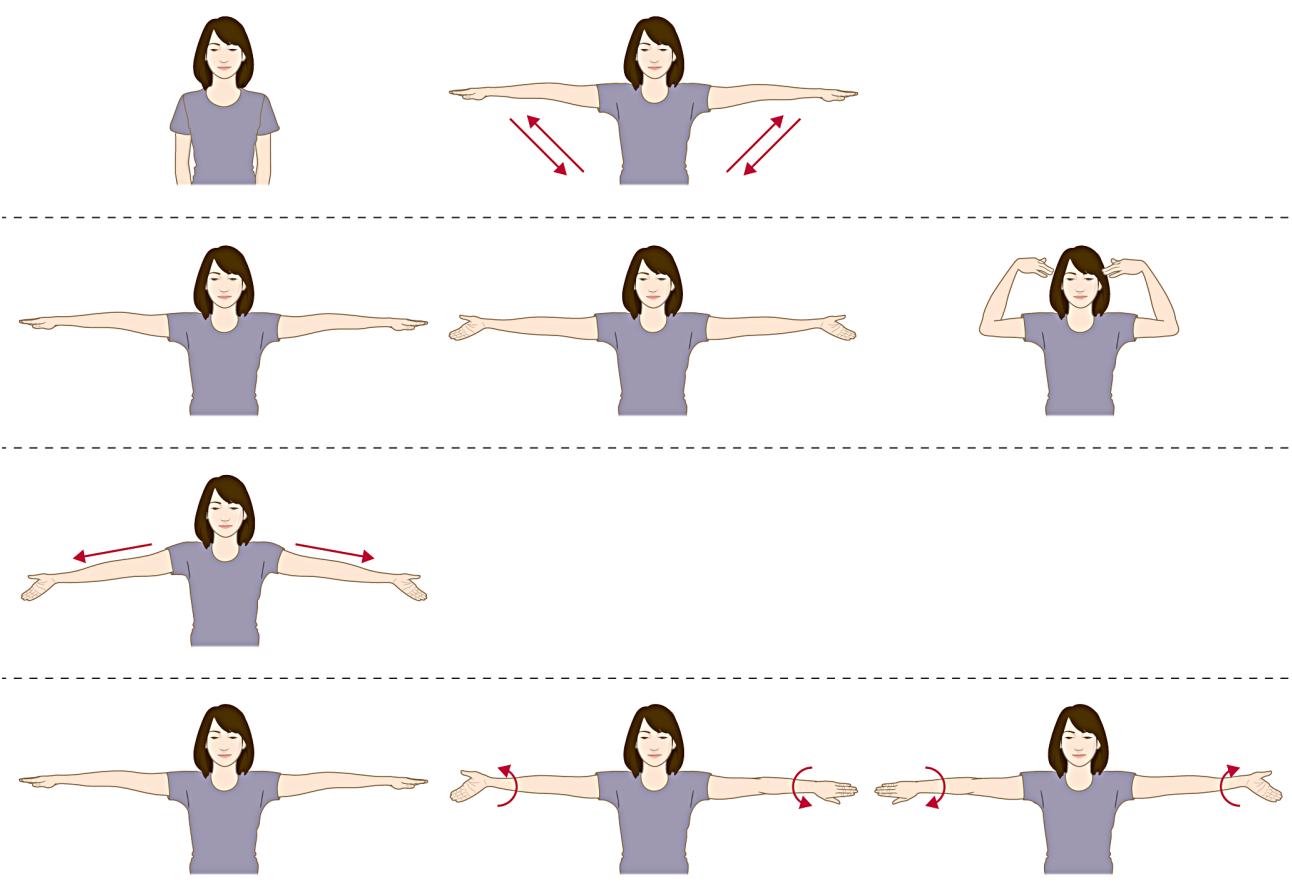

Fig. 1. Schematics of our institution's arm exercise protocol. 


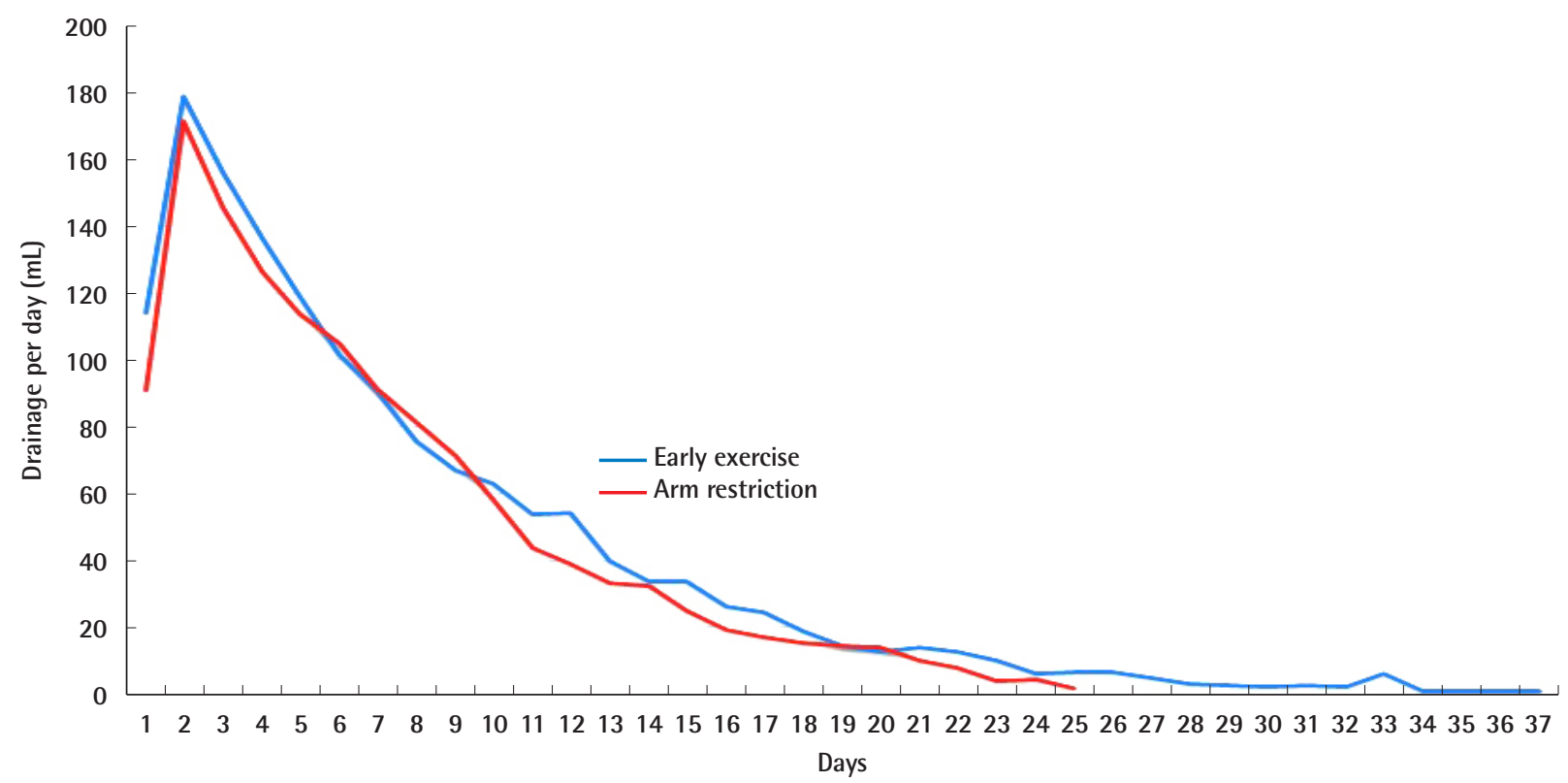

Fig. 2. The time course of daily drainage amounts. The daily drainage volume was calculated as the summation of the amounts of the two drains over a 24-hour period.
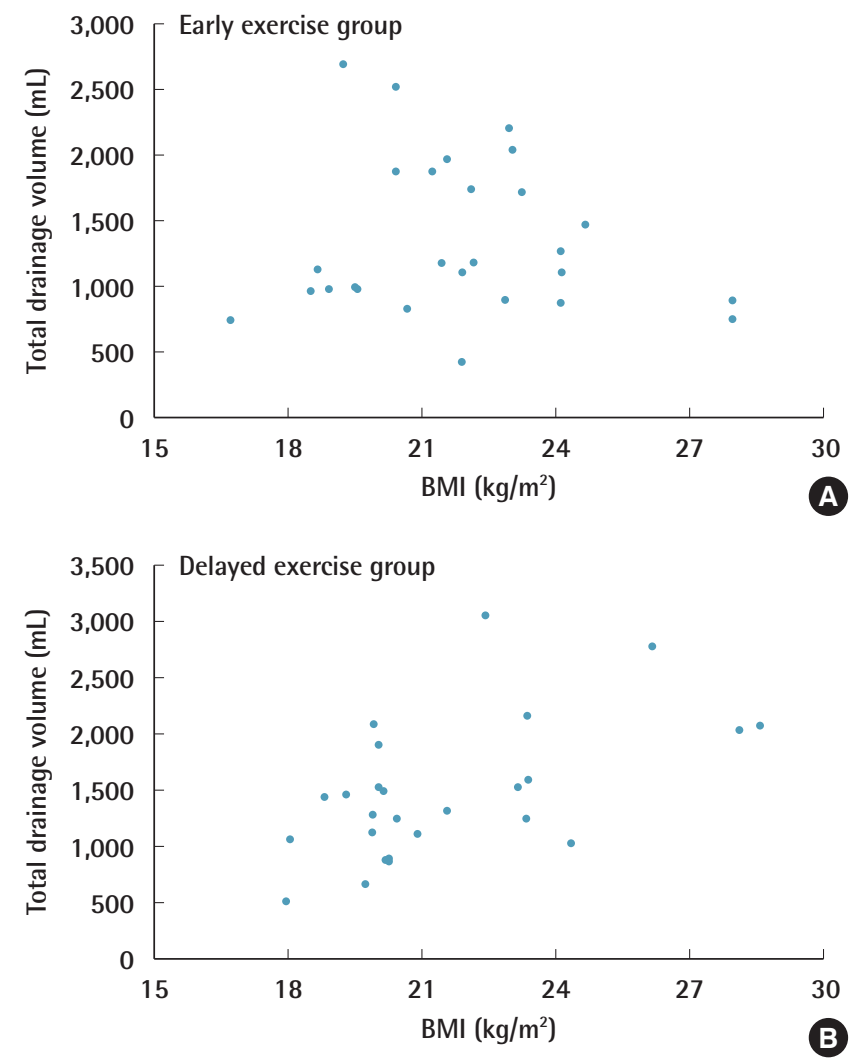

Fig. 3. Body mass index (BMI) versus the total drainage volume in each group $(A, B)$.

$\mathrm{P}=0.361$ and $\mathrm{P}=0.095$, respectively). The results were similar, as there were no significant differences between the two groups regarding the total drainage volume and the duration of the drainage maintenance period. The average total drainage volume
Table 2. Total drainage volume and maintenance period

\begin{tabular}{lccc}
\hline Variable & $\begin{array}{c}\text { Early exercise } \\
(\mathrm{n}=28)\end{array}$ & $\begin{array}{c}\text { Arm restriction } \\
(\mathrm{n}=28)\end{array}$ & P-value \\
\hline Total drainage volume $(\mathrm{mL})$ & $1,497.00 \pm 595.30$ & $1,336.00 \pm 581.82$ & 0.311 \\
POD at first drain removal & $9.36 \pm 2.79$ & $9.79 \pm 2.79$ & 0.568 \\
POD at second drain removal & $19.71 \pm 7.04$ & $17.11 \pm 4.55$ & 0.106 \\
\hline
\end{tabular}

Values are presented as the mean \pm SD.

POD, postoperative day.

Table 3. Total drainage volume and maintenance period (without ALND)

\begin{tabular}{lccc}
\hline Variable & $\begin{array}{c}\text { Early exercise } \\
(\mathrm{n}=26)\end{array}$ & $\begin{array}{c}\text { Arm restriction } \\
(\mathrm{n}=22)\end{array}$ & P-value \\
\hline Total drainage volume $(\mathrm{mL})$ & $1,457.00 \pm 558.91$ & $1,303.00 \pm 596.20$ & 0.361 \\
POD at first drain removal & $9.08 \pm 2.45$ & $9.91 \pm 2.91$ & 0.287 \\
POD at second drain removal & $19.62 \pm 6.95$ & $16.64 \pm 4.70$ & 0.095 \\
\hline
\end{tabular}

Values are presented as the mean $\pm S D$.

ALND, axillary lymph node dissection; POD, postoperative day.

Table 4. Total drainage volume and maintenance period (SLNB vs. ALND)

\begin{tabular}{lccc}
\hline Variable & $\begin{array}{c}\text { SLNB } \\
(n=48)\end{array}$ & $\begin{array}{c}\text { ALND } \\
(n=8)\end{array}$ & P-value \\
\hline Total drainage volume $(\mathrm{mL})$ & $1,387.00 \pm 575.32$ & $1,598.00 \pm 676.78$ & 0.351 \\
POD at first drain removal & $9.46 \pm 2.67$ & $10.25 \pm 3.45$ & 0.460 \\
POD at second drain removal & $18.25 \pm 6.15$ & $19.38 \pm 5.45$ & 0.607 \\
\hline
\end{tabular}

Values are presented as the mean $\pm S D$.

SLNB, sentinel lymph node biopsy; ALND, axillary lymph node dissection; POD, postoperative day.

was slightly larger in the ALND group $(1,598.00 \pm 676.78 \mathrm{~mL})$ than in the SLNB group $(1,387.00 \pm 575.32 \mathrm{~mL})$, but showed no statistically significant difference $(\mathrm{P}=0.351)$ (Table 4$)$. 
Table 5. Linear regression analysis of factors related to the total drainage volume and maintenance period

\begin{tabular}{|c|c|c|c|c|c|c|}
\hline \multirow{2}{*}{ Factor } & \multicolumn{3}{|c|}{$\begin{array}{c}\text { Univariate linear regression for } \\
\text { total drainage }\end{array}$} & \multicolumn{3}{|c|}{$\begin{array}{l}\text { Univariate linear regression for } \\
\text { drainage maintenance period }\end{array}$} \\
\hline & $\begin{array}{l}\text { Regression } \\
\text { coefficient }\end{array}$ & P-value & $\mathrm{R}^{2}$ & $\begin{array}{l}\text { Regression } \\
\text { coefficient }\end{array}$ & P-value & $\mathrm{R}^{2}$ \\
\hline Age (yr) & 24.539 & 0.011 & 0.113 & 0.135 & 0.182 & 0.033 \\
\hline Body mass index $\left(\mathrm{kg} / \mathrm{m}^{2}\right)$ & 42.380 & 0.152 & 0.038 & 0.285 & 0.349 & 0.016 \\
\hline Mastectomy specimen weight (g) & 1.290 & 0.003 & 0.155 & 0.009 & 0.035 & 0.079 \\
\hline Lymph node surgery (SLNBa)/ALND) & 211.821 & 0.351 & 0.016 & 1.125 & 0.629 & 0.004 \\
\hline Expander type (Allerganª/Mentor) & -189.396 & 0.405 & 0.013 & 1.271 & 0.585 & 0.006 \\
\hline Arm exercise (early exercise ${ }^{a} /$ arm restriction) & -160.861 & 0.311 & 0.019 & -2.607 & 0.106 & 0.048 \\
\hline
\end{tabular}

SLNB, sentinel lymph node biopsy; ALND, axillary lymph node dissection.

a) Reference group.

The univariate linear regression results are summarized in Table 5. Factors such as age and specimen weight $(\mathrm{P}<0.03)$ showed meaningful correlations with total drainage volume. However, their regression coefficients and R-squared values were too small. BMI, the type of lymph node surgery (SLNB vs. ALND), the type of expander used, and the arm exercise protocol (early exercise/arm restriction) were not associated with the total drainage volume. None of these factors affected the length of the drainage maintenance period.

\section{DISCUSSION}

Mastectomy itself can alter the motor patterns of the scapula [7]; therefore, a careful intervention to restore normal shoulder function and prevent movement impairment is necessary for these patients. Since the first report that early physical therapy led to a lower incidence of postoperative frozen shoulder, early arm exercise has been emphasized as a way of preventing shoulder morbidity in mastectomy patients.

However, many studies recommend delayed exercise postoperatively rather than an early intervention. A randomized controlled trial conducted by Abe et al. [4] showed a significant decrease in the total drainage volume and a lower incidence of seroma formation in the delayed exercise group. A different systematic review revealed that current evidence from randomized controlled trials supports the use of delayed arm exercise programs to reduce seroma formation, although clinical and statistical inconstancies exist regarding the effect of this restriction on shoulder function [8].

In contrast to these prior studies, our study showed no significant difference in the total drainage volume or the length of the drainage maintenance period between the early exercise group and the delayed exercise group. This finding may have been due to the use of tissue expanders in our study. A foreign body (such as a tissue expander) may have caused an increase in the drainage volume in both groups, which led to the discrepancy be- tween our findings and the correlations reported in earlier studies. The aforementioned studies excluded patients who underwent reconstructive surgery. In fact, previous studies have already shown that the type or size of tissue expander used can affect the amount of drainage volume postoperatively [8-10], which provides context for the finding that the total drainage volume in our study was larger than all volumes reported in previous studies. Another aspect of how our study differed from other previous reports was in its patient selection; most studies have included all patients who underwent mastectomy, regardless of the type of breast reconstruction that followed. Although a prospective study with a larger patient group may be necessary, our study's results indicate why we suggest early arm exercise rather than delayed exercise.

Some previous reports have shown that ALND is a factor that can lead to postoperative seroma or lymphedema $[11,12]$. However, there was not a substantial difference in the drainage volume between the ALND group and the SLNB group in our study. This finding may have been due to the small number of patients who underwent ALND; with a larger patient group, the results might have differed.

Although there was no statistically significant difference between the two groups, the total drainage volume was larger and the drainage maintenance period was longer in our early exercise group. There might have been a meaningful difference if the sample size had been larger. Nonetheless, the final drain removal period differed in the two groups only by a day or two. Considering the benefits and risks to patients, it seems reasonable to recommend early exercise in these patients to maintain shoulder function despite the possibility of a somewhat longer drain duration. Additional long-term research is needed to determine whether patients who engage in early exercise have better arm function.

Age showed a strong correlation with the total drainage volume in the univariate analysis $(\mathrm{P}=0.011)$. A previous study from our institution reported the same result; older patients had 
a larger drainage volume [10]. The specimen weight also demonstrated a significant correlation with the total drainage volume. However, our regression coefficient and R-squared values were relatively small, which means that further study is necessary to identify a meaningful relationship.

Surgical techniques have also advanced. First, it has not been long since $\mathrm{ADMs}$ were routinely used in expander-based reconstruction cases; the presence of $\mathrm{ADMs}$ might have influenced drainage volume in both groups. Secondly, as of 2020, the authors prefer prepectoral insertion of expanders. This study was conducted in 2018. In the authors' opinion, subpectoral positioning of the tissue expander does not seem to affect the final range of motion or activity of patients, although a few patientsespecially those who receive postoperative radiotherapy-suffer unnatural discomfort in shoulder motion. Further studies need to explore these factors in greater depth to achieve a standardized exercise protocol.

In this study, arm movement restrictions did not produce a significant difference in the drainage volume or duration of drain placement. In conclusion, to prevent arm and shoulder morbidity, we recommend early rather than delayed exercise in mastectomy patients who undergo immediate tissue expanderbased breast reconstruction. Further research that involves the long-term follow-up of expander-based reconstruction patients regarding their shoulder-related morbidity, complications (including seroma formation), infection rates, and incidence of contracture will be necessary to determine optimal arm exercise-related guidelines.

\section{NOTES}

\section{Conflict of interest}

Won Jai Lee and Seung Yong Song are editorial board members of the journal but were not involved in the peer reviewer selection, evaluation, or decision process of this article. No other potential conflicts of interest relevant to this article were reported.

\section{Ethical approval}

The study was approved by the Institutional Review Board of Severance Hospital (IRB No. 4-2017-1054) and performed in accordance with the principles of the Declaration of Helsinki. Written informed consent was obtained.

\section{Author contribution}

Conceptualization: DW Lee, SY Song. Formal analysis: OY Joo, SJ Moon. Methodology: OY Joo, DH Lew. Project administration: WJ Lee, SY Song. Visualization: OY Joo. Writing - original draft: OY Joo. Writing - review \& editing: OY Joo, SY Song.

\section{Data availability statement}

The data that support the findings of this study are available from the corresponding author upon reasonable request.

\section{ORCID}

Oh Young Joo https://orcid.org/0000-0001-5736-4671

Seung Jin Moon

Dong Won Lee

Dae Hyun Lew

Won Jai Lee

Seung Yong Song https://orcid.org/0000-0003-1980-0270

https://orcid.org/0000-0003-0046-3139

https://orcid.org/0000-0002-2625-5664

https://orcid.org/0000-0003-3056-0503

https://orcid.org/0000-0002-3145-7463

\section{REFERENCES}

1. Hack TF, Kwan WB, Thomas-Maclean RL, et al. Predictors of arm morbidity following breast cancer surgery. Psychooncology 2010;19:1205-12.

2. Sclafani LM, Baron RH. Sentinel lymph node biopsy and axillary dissection: added morbidity of the arm, shoulder and chest wall after mastectomy and reconstruction. Cancer J 2008;14:216-22.

3. Bendz I, Fagevik Olsen M. Evaluation of immediate versus delayed shoulder exercises after breast cancer surgery including lymph node dissection: a randomised controlled trial. Breast 2002;11:241-8.

4. Abe M, Iwase T, Takeuchi T, et al. A randomized controlled trial on the prevention of seroma after partial or total mastectomy and axillary lymph node dissection. Breast Cancer 1998;5:67-9.

5. Xue DQ, Qian C, Yang L, et al. Risk factors for surgical site infections after breast surgery: a systematic review and metaanalysis. Eur J Surg Oncol 2012;38:375-81.

6. de Haan A, Toor A, Hage JJ, et al. Function of the pectoralis major muscle after combined skin-sparing mastectomy and immediate reconstruction by subpectoral implantation of a prosthesis. Ann Plast Surg 2007;59:605-10.

7. Crosbie J, Kilbreath SL, Dylke E, et al. Effects of mastectomy on shoulder and spinal kinematics during bilateral upper-limb movement. Phys Ther 2010;90:679-92.

8. Shamley D, Lascurain-Aguirrebena I, Oskrochi R, et al. Shoulder morbidity after treatment for breast cancer is bilateral and greater after mastectomy. Acta Oncol 2012;51: 1045-53.

9. Lee KT, Hong SH, Jeon BJ, et al. Predictors for prolonged drainage following tissue expander-based breast reconstruction. Plast Reconstr Surg 2019; 144:9e-17e.

10. Lim YM, Lew DH, Roh TS, et al. Analysis of factors that affect drainage volume after expander-based breast recon- 
struction. Arch Plast Surg 2020;47:33-41.

11. Hashemi E, Kaviani A, Najafi M, et al. Seroma formation after surgery for breast cancer. World J Surg Oncol 2004;2:44.

12. Del Bianco P, Zavagno G, Burelli P, et al. Morbidity compar- ison of sentinel lymph node biopsy versus conventional axillary lymph node dissection for breast cancer patients: results of the sentinella-GIVOM Italian randomised clinical trial. Eur J Surg Oncol 2008;34:508-13. 